01

\title{
Влияние плотности атомов буферного газа на форму эксимерной полосы $\mathrm{HgXe}\left(A^{3} 0^{+}\right) \rightarrow \operatorname{HgXe}\left(X^{1} 0^{+}\right)$
}

\author{
(C) А.З. Девдариани ${ }^{1,3}$, А.Л. Загребин ${ }^{2}$, Н.А. Крюков ${ }^{1}$, \\ М.Г. Леднев ${ }^{2}$, Н.А. Тимофеев ${ }^{1}$ \\ ${ }^{1}$ Санкт-Петербургский государственный университет, \\ 198504 Санкт-Петербург, Россия \\ ${ }^{2}$ Балтийский государственный технический университет „ВОЕНМЕХ“ им. Д.Ф. Устинова, \\ 190005 Санкт-Петербург, Россия \\ ${ }^{3}$ Российский государственный педагогический университет им. А.И. Герцена, \\ 191186 Санкт-Петербург, Россия \\ e-mail: snbrn2@yandex.ru, alzagrebin@yandex.ru, nkryukov@yandex.ru,m_lednev@rambler.ru, niktimof@yandex.ru
}

Поступила в редакцию 24.12.2018 г.

В окончательной редакции 24.12.2018 г.

Принята к публикации 09.01.2019 г.

\begin{abstract}
Проанализированы причины расхождений в экспериментальных данных по форме длинноволновых континуумов вблизи резонансной линии $6^{3} P_{1} \rightarrow 6{ }^{1} S_{0}$ атома ртути в атмосфере ксенона, полученных разными авторами. Расчеты спектральных профилей для предельных случаев высокой и низкой плотностей атомов буферного газа позволяют сделать вывод о том, что расхождения вызваны, главным образом, зависимостью скоростей трехчастичной рекомбинации и релаксационных процессов от плотности ксенона. При различных скоростях этих процессов формируются разные типы неравновесной или почти равновесной (при высоких концентрациях ксенона) колебательно-вращательной заселенности состояний эксимерной молекулы $\mathrm{HgXe}\left(A^{3} 0^{+}\right)$, что и влияет на спектральные профили излучения.
\end{abstract}

DOI: $10.21883 /$ OS.2019.05.47648.369-18

\section{Введение}

Оптическая спектроскопия эксимеров была инициирована работами по созданию эксимерных лазеров [1]. Для таких молекул $\left(\mathrm{M}^{*} \mathrm{X}\right)$, составленных при больших межатомных расстояниях из атома металла $\mathbf{M}^{*}$ в нижних излучающих состояниях и атома инертного газа $\mathrm{X}$ в основном состоянии, особый интерес представляет формирование и излучение эксимерных полос в крыльях линий паров металлов в видимой или УФ, ВУФ и ИК областях спектра [2]. Типичный пример эксимерная полоса, которая формируется при переходе $\operatorname{HgXe}\left(A^{3} 0^{+}\right) \rightarrow \operatorname{HgXe}\left(X^{1} 0^{+}\right)$. Несмотря на сравнительную доступность спектра для экспериментального изучения, опубликованные данные [3-5] при температуре $T \sim 300 \mathrm{~K}$ не только различаются, но и отсутствует убедительное обсуждение причин расхождений.

В настоящей работе показано, что расхождения обусловлены в основном зависимостью скоростей рекомбинационных и релаксационных процессов от концентрации атомов ксенона, что приводит к формированию различных (при разных концентрациях атомов буферного газа) типов неравновесной или почти равновесной заселенности колебательно-вращательных состояний эксимерной молекулы $\operatorname{HgXe}\left(A^{3} 0^{+}\right)$.

Предварительные результаты работы доложены на 23-й Международной конференции по форме спектральных линий [6].

\section{Профили крыльев спектральных линий эксимеров}

Форма линии оптического перехода между двумя квазимолекулярными состояниями, которые формируются при столкновениях атомов, определяется зависимостью потенциалов взаимодействия в начальном и конечном состояниях от межатомного расстояния $R$ [7]. Для расчетов этих характеристик были развиты полуэмпирические методы описания взаимодействия атомов $[8,9]$.

В случае эксимеров спектр полосы в общем случае формируется переходами из связанных $(b)$ и квазисвязанных $(q b)$ состояний движения атомов, а также переходами $(c)$ из состояний свободного движения атомов в терме начального состояния. Поэтому полный вклад соответствующих переходов в спектральное распределение интенсивности молекулярной полосы помимо термов определяется также и заселенностью начальных состояний.

В зависимости от экспериментальных условий между начальными состояниями устанавливается (или отсутствует) термодинамическое равновесие, поэтому вычислить в общем виде форму полосы для произвольного давления буферного газа и произвольной температуры не представляется возможным. В важном частном случае максвелл-больцмановского распределения атомов профиль крыла спектральной линии может быть 
представлен [10] в виде

$$
\begin{aligned}
I(\lambda) \sim & \frac{1}{\lambda^{3}} \frac{D^{2}\left(R_{c}\right) R_{c}^{2}}{\left|\frac{d}{d R} \Delta U_{\mathrm{if}}\left(R_{C}\right)\right|} \\
& \times\left\{\Phi_{b}\left(T, R_{c}\right)+\Phi_{q b}\left(T, R_{c}\right)+\Phi_{c}\left(T, R_{c}\right)\right\},
\end{aligned}
$$

где $D\left(R_{C}\right)$ - дипольный момент оптического перехода, $R_{c}$ - так называемая кондоновская точка, которая определяется из условия

$$
\Delta U_{i f}\left(R_{c}\right)=U_{i}\left(R_{c}\right)-U_{f}\left(R_{c}\right)=2 \pi \hbar c / \lambda,
$$

$U_{i, f}$ - потенциалы взаимодействия атомов в начальном возбужденном и конечном основном состояниях, отсчитанные от энергии невзаимодействующих атомов в основных состояниях, $\Phi_{b, q b, c}$ - вклады соответствующих состояний движения атомов.

\section{Профиль крыла спектральной линии в предельных случаях высокого и низкого давлений буферного газа}

Отсутствие данных по элементарным процессам, которые участвуют в формировании заселенностей различных состояний эксимера, не позволяет вычислить спектральные профили, определенные при различных экспериментальных условиях [3-5]. Тем не менее, возможно получить представление о влиянии заселения состояний с помощью подходов, использованных в [10-12] для оценки влияния плотности буферного газа на спектры возбужденных квазимолекул.

С этой целью выполнен расчет спектральных профилей в двух предельных случаях: для высокой концентрации атомов буферного газа, когда заселение состояний эксимера равновесное, и низкой концентрации атомов буферного газа, когда спектр формируется в основном оптическими переходами при парных столкновениях атомов с максвелловским распределением по скоростям. В случае высокой концентрации атомов буферного газа расчеты выполнены в рамках квазистатического подхода [7]. В случае низкой концентрации, когда связанные и квазисвязанные состояния отсутствуют и радиационные переходы происходят только при парных столкновениях, для расчета профиля использовался подход [11], который позволяет учесть усложнения, связанные с существованием центробежного барьера при классическом движении атомов в немонотонном эффективном потенциале. Достоинство используемых подходов в том, что они позволяют оценить форму контура без привлечения данных по элементарным процессам трехчастичной атомной рекомбинации и релаксации.

В расчетах использованы восстановленный из экспериментальных данных по температурной зависимости коэффициента поглощения [13] потенциал взаимодействия в возбужденном состоянии $A^{3} 0^{+}$и потенциалы

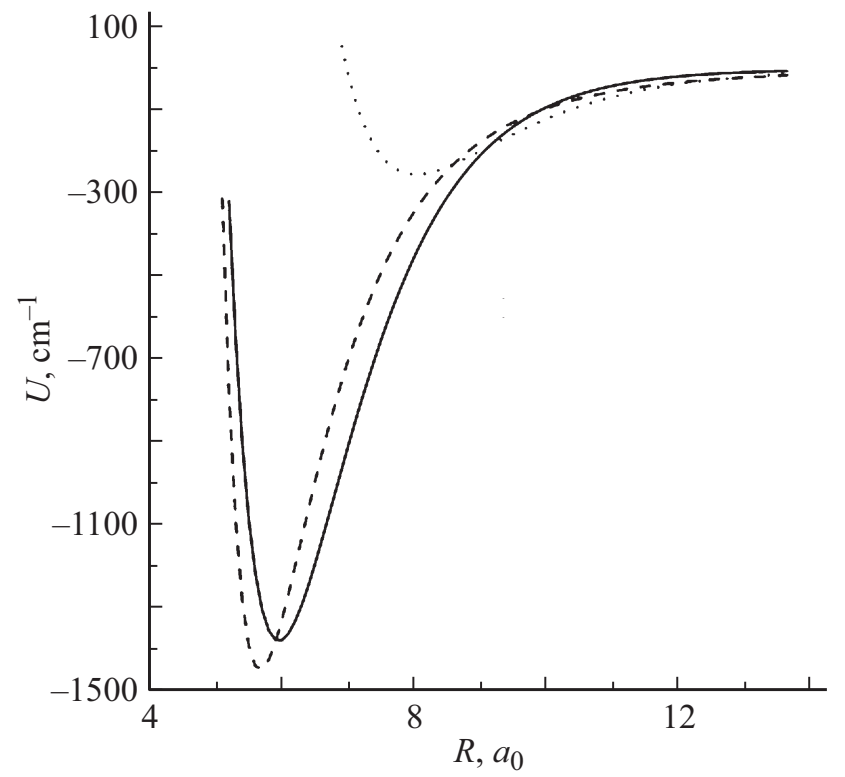

Рис. 1. Потенциальные кривые взаимодействия для возбужденного состояния $A^{3} 0^{+}$, полученные в [13] (штриховая линия) и [14] (сплошная линия), а также потенциальная кривая для основного состояния $X^{1} 0^{+}$[14] (пунктирная линия).

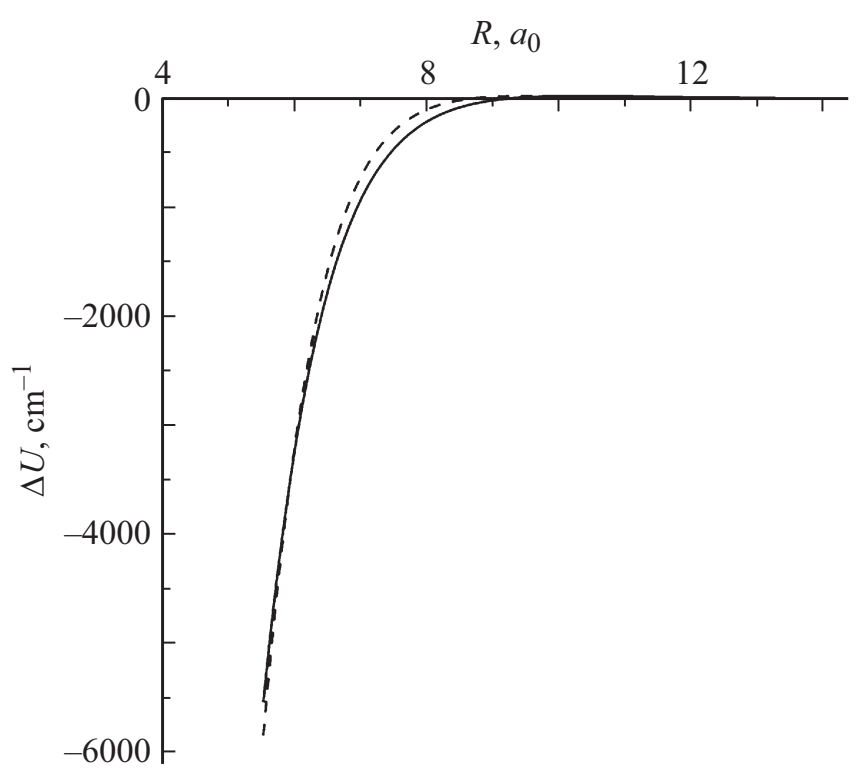

Рис. 2. Разностные потенциалы $\Delta U=U\left(A^{3} 0^{+}\right)-U\left(X^{1} 0^{+}\right)$: штриховая кривая - для основного и возбужденного состояний использованы данные работ $[13,14]$, сплошная кривая по данным работы [14].

взаимодействия в возбужденном и основном состояниях в форме Морзе [14], которые определены из экспериментов по лазерно-индуцированной флуоресценции (рис. 1). Соответствующие разностные потенциалы $\Delta U_{i f}$ приведены на рис. 2.

Экспериментальные спектры излучения [3-5], полученные при различных значениях концентраций атомов ксенона $N_{\text {Хе }}$ и рассчитанные в приближениях высокой и 


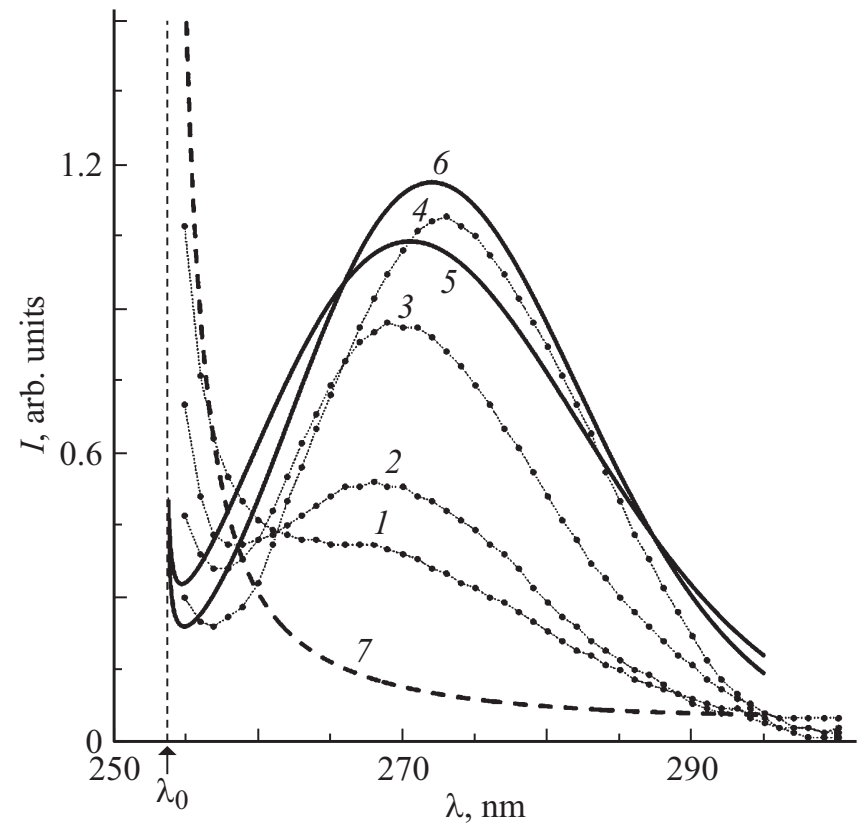

Рис. 3. Экспериментальные (1-4) и расчетные (5-7) спектральные профили излучения в относительных единицах. $N_{\mathrm{Xe}}=3 \cdot 10^{17} \quad(1) \quad[3], \quad 10.1 \cdot 10^{17} \quad$ (2) [3], $10^{19}$ (3) [4], $1.1 \cdot 10^{20} \mathrm{~cm}^{-3}(4)[5] .5$ - расчет с использованием потенциалов для состояний $A^{3} 0^{+}$и $X^{1} 0^{+}$из работ $[13,14]$ соответственно, 6 - расчет с использованием потенциалов возбужденного и основного состояний, приведенных в работе [14]. 5,6 расчет в приближении высокой концентрации атомов Хе. 7 - расчет в приближении низкой концентрации атомов Хе с использованием потенциалов для состояний $A^{3} 0^{+}$и $X^{1} 0^{+}$ из работ $[13,14]$ соответственно. $\lambda_{0}=253.7 \mathrm{~nm}-$ положение резонансной линии $6^{3} P_{1}-6^{1} S_{0}$.

низкой концентрации атомов ксенона, представлены на рис. 3.

\section{Заключение}

Сопоставление результатов расчетов профиля крыла спектральной линии, который формируется при переходе $\operatorname{HgXe}\left(A^{3} 0^{+}\right) \rightarrow \operatorname{HgXe}\left(X^{1} 0^{+}\right)$в предельных случаях высокого и низкого давлений атомов Хе, с экспериментальными данными при различных давлениях позволяет заключить, что в основе расхождений экспериментальных данных лежит конкуренция скоростей оптических переходов при столкновениях атомов в квазимолекулярном состоянии $A^{3} 0^{+}$и рекомбинации атомов $\operatorname{Hg}\left({ }^{3} P_{1}\right)$ и $\mathrm{Xe}\left({ }^{1} S_{0}\right)$ в эксимер $\operatorname{HgXe}\left(A^{3} 0^{+}\right)$с последующей релаксацией возбужденных колебательных состояний и излучением из нижерасположенных связанных состояний. При плотностях атомов буферного газа менее $3 \cdot 10^{17} \mathrm{~cm}^{-3}$ крыло спектральной линии $\operatorname{Hg}\left({ }^{3} P_{1} \rightarrow{ }^{1} S_{0}\right)$ в диапазоне $\lambda \sim 255-280 \mathrm{~nm}$ формируется в ходе парных столкновений атомов $\operatorname{Hg}\left({ }^{3} P_{1}\right)$ и атомов Хе. При плотностях $N_{\text {Хе }}>10^{20} \mathrm{~cm}^{-3}$ крыло спектральной линии формируется переходами из почти равновесно заселенных колебательно-вращательных состояний.

Хотя первоначально интерес к спектроскопии эксимеров определялся лазерной тематикой, в последние годы спектроскопия эксимеров востребована и в работах по созданию источников сплошного излучения. При этом особо интересны смеси с ксеноном, так как по сравнению с другими инертными газами для таких гетерои гомоядерных эксимеров характерно наличие сравнительно мощной потенциальной ямы и, как следствие, более широкополосный спектральный диапазон излучения. Перспективными оказываются не только эксимеры с атомами металлов, но также эксимеры с атомами галогенов [15], гетероядерные [16] и гомоядерные [17] эксимеры атомов инертных газов.

\section{Список литературы}

[1] Basting D., Marowsky G. (Eds.) Excimer Laser Technology. Springer, 2005. $434 \mathrm{p}$.

[2] Лисицына Н.В. Эксимерные лазеры / Учебное пособие. М.: МГТУ им. Н.Э. Баумана, 2006. 28 с.

[3] Крюков Н.А. // Опт. и спектр. 1989. Т. 66. В. 4. С. 721.

[4] Lam L.K., Gallagher A., Drulligher R. // J. Chem. Phys. 1978. V. 68. P. 4411.

[5] Никифоров Е.В. // Опт. и спектр. 1976. Т. 41. В. 1. С. 339.

[6] Devdariani A.Z., Grigorian G.M., Kryukov N.A., Lednev M.G., Zagrebin A.L. // J. Phys.: Conf. Series. 2017. V. 810. P. 012028.

[7] Девдариани А.3. // Опт. и спектр. 1999. Т. 86. № 6. С. 954; Devdariani A.Z. // Opt. Spectrosc. 1999. V. 86. N 6. P. 853.

[8] Загребин А.Л., Леднев М.Г. // Опт. и спектр. 1999. Т. 87. № 6. C. 893; Zagrebin A.L., Lednev M.G. // Opt. Spectrosc. 1999. V. 86. N 6. P. 812.

[9] Загребин А.Л., Леднев М.Г. // Опт. и спектр. 1995. Т. 78. № 2. C. 183; Zagrebin A.L., Lednev M.G. // Opt. Spectrosc. 1995. V. 78. N 2. P. 159.

[10] Беляев А.К., Девдариани А.З., Себякин Ю.Н. // Опт. и спектр. 1985. Т. 59. В. 3. С. 505; Belyaev A.K., Devdariani A.Z., Sebyakin Yu.N. // Opt. Spectrosc. 1985. V. 59. N 3. P. 305.

[11] Девдариани А.З., Загребин А.Л. // Опт. и спектр. 1992. T. 72. B. 3. C. 571; Devdariani A.Z., Zagrebin A.L. // Opt. Spectrosc. 1992. V. 72. N 3. P. 309.

[12] Загребин А.Л., Себякин Ю.Н. // Опт. и спектр. 1993. Т. 74. B. 4. C. 667.

[13] Grycuk T., Findeisen M. // J. Phys. B. 1983. V. 16. P. 975.

[14] Okunishi M., Nakazava H., Yamanouchi K., Tsuchiya S. // J. Chem. Phys. 1990. V. 93. P. 7526.

[15] Головицкий А.П. // ЖТФ. 2011. Т. 81. № 3. С. 45.

[16] Герасимов Г.Н. // УФН. 2004. Т. 174. В. 2. С. 155.

[17] Lisenko A.A., Lomaev M.I., Skakun V.S., Tarasenko V.F. // Phys. Scr. 2007. V. 76. P. 211. 